\title{
Adverse outcomes after surgery in patients with previous stroke
}

C.C. $\mathrm{Liao}^{1,2,3}$, P.Y. Chang ${ }^{4}$, C.C. $\mathrm{Yeh}^{5,8}$, C.J. $\mathrm{Hu}^{3,6}$, C.H. Wu ${ }^{3,7}$, T.L. Chen ${ }^{1,2,3}$

${ }^{1}$ Department of Anesthesiology, Taipei Medical University Hospital, Taipei, Taiwan

${ }^{2}$ Health Policy Research Center, Taipei Medical University Hospital, Taipei, Taiwan

${ }^{3}$ School of Medicine, Taipei Medical University, Taipei, Taiwan

${ }^{4}$ Department of Eldercare, National Tainan Institute of Nursing, Tainan City, Taiwan

${ }^{5}$ Department of Surgery, China Medical University Hospital, Taichung, Taiwan

${ }^{6}$ Department of Neurology, Shuang Ho Hospital, Taipei Medical University, Taipei, Taiwan

${ }^{7}$ Department of Surgery, Shuang Ho Hospital, Taipei Medical University, Taipei, Taiwan

${ }^{8}$ Department of Surgery, University of Illinois, Chicago, Illinois, USA

Drs. CH Wu and TL Chen contributed equally.

\section{Corresponding author / address reprint requests to:}

Ta-Liang Chen, MD, PhD

Professor and Director

Department of Anesthesiology

Taipei Medical University Hospital

252 Wuxing St., Taipei 11031, Taiwan

Tel: 886-2-2737-2181, ext. 8310

Fax: 886-2-2736-7344 
E-mail: tlc@tmu.edu.tw

Word counts: Abstract, 260 words; Text, 1769 words

Tables: 5; References: 30

Keywords: stroke, surgery, postoperative complications, mortality

Sources of funding: This work was supported by the National Science Council, Taiwan

(NSC102-2314-B-038-021-MY3).

\section{List of abbreviations}

CI, confidence interval

$\mathrm{RR}$, rate ratio

ICD-9-CM, International Classification of Diseases, 9th Revision, Clinical Modification

ICU, intensive care unit

Competing Interests: The authors have declared that no competing interests exist.

Contributors: Conceived and designed the experiments: CCL PYC CCY CJH CHW TLC.

Analyzed the data: CCL. Wrote the first draft of the manuscript: CCL. Contributed to the writing of the manuscript: CCL PYC CCY CJH CHW TLC. ICMJE criteria for authorship read and met: CCL PYC CCY CJH CHW TLC. Agree with manuscript results and conclusions: CCL PYC CCY CJH CHW TLC. 


\begin{abstract}
Background: Limited information is available on the association between a medical history of stroke and postoperative outcomes. This study aimed to investigate the outcomes after non-neurological surgeries in patients with previous stroke.
\end{abstract}

Methods: Using Taiwan's National Health Insurance Research Database, a nationwide cohort study was conducted consisting of patients who underwent non-neurological surgeries 2008 to 2010 with a medical history of stroke within the 24-month period prior to surgery. Patients who underwent non-neurological surgeries without previous stroke were selected as controls by the propensity score-matched pair method. The 30-day postoperative complications and in-hospital mortality were compared between the groups of patients.

Results: There were 1426795 adults who underwent major inpatient non-neurological surgeries and 45420 of them had previous stroke. Patients that underwent surgeries with previous stroke had increased risk of postoperative pneumonia, septicemia, acute renal failure, acute myocardial infarction, pulmonary embolism, and 30-day in-hospital mortality, adjusted rate ratio (RR) 1.79 (95 per cent confidence interval (c.i.) 1.61 to 1.99). Compared with controls, patients with previous stroke due to intracerebral hemorrhage, RR 3.41 (2.97 to 3.91) and who were treated in an intensive care unit intensive care, RR 2.55 (2.24 to 2.90), or received neurosurgery, RR 2.49 (2.12 to 2.92 ) had an increased 30-day in-hospital mortality. Postoperative mortality also increased with preoperative stroke related comorbidities, and a 
medical history of stroke within 1-6 months prior to surgery, RR 3.31 (2.91 to 3.75).

Conclusions: Patients with previous stroke had a higher risk for postoperative adverse outcome with a 30-day in-hospital mortality nearly twofold that of patients without previous stroke. 


\section{Introduction}

Although the incidence of stroke has been declining ${ }^{1}$, stroke remains the worldwide leading cause of acquired disability and death in adults ${ }^{2-4}$. Risk factors, prevention strategies and genetic biomarkers for stroke have been studied extensively ${ }^{3}$. Rehabilitation, such as physical therapy, may prevent squealae after stroke and improve functional status ${ }^{5}$. However, patients with previous stroke still suffer from the common sequelae and complications such as dementia, depression, pneumonia, urinary tract infection, decubitus ulcer, hip fracture, traumatic brain injury, epilepsy, dysphagia and constipation ${ }^{5-8}$. Thus, the acute care services for patients with previous stroke have been documented with higher complication and mortality rates $6,7,9$

Studies investigating postoperative adverse events in patients with previous stroke have been limited by small sample sizes ${ }^{10-12}$, single type of surgical procedure ${ }^{10-16}$, lack of control $\operatorname{group}^{10-19}$, without matching ${ }^{10-19}$, inadequate adjustment for potential confounding effects ${ }^{10,14}$ and reporting single outcome $\mathrm{e}^{10-12,15}$.

By the use of Taiwan's National Health Insurance Research Database, a population-based, cohort study was conducted to investigate the full spectrum of postoperative adverse outcomes and analyze the impact of disease severity for patients with previous stroke that received non-neurological major surgeries. 


\section{Methods}

\section{Source of data}

The reimbursement claims data from Taiwan's National Health Insurance were used in this study. More than 99 per cent of the 22.6 million Taiwan residents are enrolled in this system. Taiwan's National Health Research Institutes established a National Health Insurance Research Database recording all in- and outpatient medical services of beneficiaries, including patient demographics, primary and secondary diagnoses of diseases, procedures, prescriptions and medical expenditures ${ }^{5,20-25}$. The accuracy of major diagnosis codes in the National Health Insurance Research Database was validated in the previous study ${ }^{26}$.

\section{Ethical approval}

Insurance reimbursement claims from Taiwan's National Health Insurance Research Database are maintained by the National Health Research Institutes and are available for public access. To protect personal privacy, the electronic database was de-identified with patient identifications scrambled for further public access for research. According to National Health Research Institutes regulations, informed consent is not required due to decoded and scrambled patient identification. However, this study was evaluated and approved by Taiwan's National Health Research Institutes. This study was conducted in accordance with the Helsinki Declaration.

\section{Study design}


From the medical claims of National Health Insurance Research Database, this study identified adults aged 20 years and above who underwent major inpatient non-neurological surgeries from 2008 to 2010. Patients underwent non-neurological surgeries defined as surgeries requiring general, epidural or spinal anesthesia and hospitalization for more than one day and who had a history of stroke within the 24-month period prior to surgery were included in the study. The criteria for identifying patients with a medical history of stroke were defined in the previous studies ${ }^{5,20,21}$.

This study used propensity score matched-pair method to select control patients without previous stroke. The propensity score was estimated using a logistic regression model including covariates generally considered to be associated with adverse events after surgery. The selected matching factors were based the previous surgical studies ${ }^{22-25}$.

\section{Definitions}

The information of operation in teaching hospital and low-income status was identified according to Bureau of National Health Insurance regulations. Low income was defined as patients qualified for waived medical copayment as certified by the Bureau of National Health Insurance. The age of study population was defined when the index surgery occurred. According to the International Classification of Diseases, 9th Revision, Clinical Modification (ICD-9-CM), stroke was defined as ICD-9-CM 430-438 in this study. Coexisting medical conditions that were considered (Suppl.Table 1) included hypertension, diabetes, mental 
disorders, chronic obstructive pulmonary diseases, ischemic heart disease, hyperlipidemia, and liver cirrhosis (ICD-9-CM 571.2 and 571.5) that were diagnosed within the 24-month period prior to surgery. Renal dialysis was also identified as a coexisting medical condition in this study.

Acute myocardial infarction, acute renal failure, deep wound infection, pneumonia, postoperative bleeding, pulmonary embolism, and septicemia, were identified as major postoperative complications occurring within the 30-day period after the index surgeries; patients who were diagnosed with these conditions during the 24-month prior to surgery were excluded from analysis. Medical resource utilization such as length of hospitalization, admission to intensive care unit (ICU) within 30 days after the index surgery and in-hospital medical expenditures were analyzed. The lengths of stay and in-hospital medical expenditures of patients underwent surgeries with and without previous stroke were categorized by dividing into quartiles. Patients who underwent surgeries within the highest quartile of length of stay or in-hospital medical expenditures among the total number of patients were defined as the individuals having increased length of stay or elevated medical expenditures.

To explore the correlation between the severity of stroke and postoperative adverse outcomes, the type of treatment for stroke, the utilization of medical resources and medical expenditures were considered for further analysis. Stroke-related comorbidities such as traumatic brain injury, dementia, pneumonia and decubitus ulcer were also identified as 
clinical indicators of stroke severity ${ }^{6-8}$.

\section{Statistical analysis}

To reduce confounding influence of covariates, a propensity score was used to match age, sex, low-income status, coexisting medical conditions, operation in teaching hospital or not, types of surgery and anesthesia between patients with and without previous stroke. The chi-square tests were used to examine the difference of parameters between patients underwent surgeries with and without previous stroke.

Adjusted rate ratios (RR) with 95 per cent confidence interval (c.i.) for 30-day postoperative complications, in-hospital mortality, prolonged length of stay, ICU stay and elevated medical expenditure between patients underwent surgeries with or without stroke were analyzed with multivariable Poisson regression by including age, sex, low-income status, operation in teaching hospital or not, preoperative coexisting medical conditions and types of surgery and anesthesia. To assess the impact of stroke severity on 30-day postoperative in-hospital mortality, multivariable Poisson regression was used to control the potential confounders; stroke-related neurosurgery, stroke within 1-6 months preoperatively, ICU stay, medical expenditure, traumatic brain injury, dementia, pneumonia and decubitus ulcer.

Differences between groups were considered significant if two-sided $P$ value was smaller than 0.05. Statistical analysis was performed with SAS, software version 9.1 (SAS Institute Inc., Cary, North Carolina, USA). 


\section{Results}

Among 1426795 patients underwent major non-neurological surgeries, 45420 of them had previous stroke. There were significant differences between patients underwent surgeries with and without previous stroke (Table 1).

After propensity score-matching (Table 2), there were no significant differences between patients underwent surgeries with and without previous stroke in terms of socio demographic variables, operation in teaching hospital, type of surgery, types of anesthesia, and medical conditions.

Before propensity score-matching (Table 3), previous stroke was associated with postoperative pneumonia, septicemia, acute renal failure, acute myocardial infarction, pulmonary embolism any complications, prolonged hospital stay, ICU stay, increased medical expenditure and 30-day in-hospital mortality. After propensity-score matching and multivariable adjusting, patients with previous stroke had higher risk of postoperative 30-day in-hospital mortality, RR 1.79, prolonged hospital stay, RR 1.40, ICU stay, RR 1.24, and increased medical expenditure, RR 1.19 as well as postoperative complications including pneumonia, septicemia, acute renal failure, acute myocardial infarction, and pulmonary embolism (Table 3). Compared with control patients (Table 4), patients with stroke within 1-6 months prior to surgery had an increased 30-day postoperative in-hospital mortality (RR 3.31). Furthermore, patients with previous stroke and with low income had an increased 30-day 
postoperative in-hospital mortality compared with patients without previous stroke.

Several indicators of stroke severity were associated with 30-day in-hospital mortality, including: stroke due to intracerebral hemorrhage, ICU treatment for stroke, stroke related neurosurgery, and high stroke-related medical expenditure. Preoperative stroke-related comorbidities increased postoperative mortality including traumatic brain injury (RR 2.01), dementia (RR 2.10), pneumonia (RR 2.16) and decubitus ulcer (RR 2.35). 


\section{Discussion}

Stroke per se has been identified as one of the major risk factors for postoperative mortality and complications. The results of the present study, which was designed with propensity score-matching and adjusted by multivariable regression models, validated the increased rates of surgical adverse events in patients with a medical history of stroke prior to surgery. The risk of postoperative mortality for patients in the propensity score-matched cohort was lower than in the non-matched cohort. This means that the risk of postoperative mortality would be overestimated if propensity score-matching had not been used, even if multivariable regression analysis had been performed to control for confounders. The matched study design with propensity score should therefore be considered for outcomes studies ${ }^{27-29}$.

The postoperative incidence of pneumonia, sepsis and acute renal failure increased remarkably in patients with previous stroke, as were myocardial infarction and pulmonary embolism, albeit to a lesser extent.

The present study considered stroke-related characteristics, treatments and complications as the clinical indicators for the severity of stroke, and dementia, traumatic brain injury, pneumonia and decubitus ulcer were highly correlated with postoperative adverse outcomes in patients with previous stroke ${ }^{6-8}$. Stroke within 1-6 months prior to surgery was associated with a more than threefold increased 30-day postoperative in-hospital mortality. Patients with a medical history of hemorrhagic stroke, ICU stay, neurosurgery for stroke and patients within 
the highest quartile of preoperative medical expenditure before surgery had an increased 30-day mortality rate. These findings show a severity-dependent association between stroke and postoperative in-hospital mortality.

Stroke is a manifestation of vascular disease that may attribute to more complications and subsequent mortality after surgery. There are, however, several other possible reasons for worse outcomes in patients with a medical history of stroke, including limited physical function, reduced pain sensitivity and mental dysfunction; the two latter may delay diagnosis and treatment of complications ${ }^{25}$. Overdosing or under dosing related to interactions between analgesics, anesthetics and patients' regular medications may occur ${ }^{25,30}$. Socioeconomic factors such as lack of family support and geographic challenges might hinder some patients from seeking medical services, and this may affect quality of care.

Several limitations in the present study are acknowledged. First, the database lacked detailed socio demographic variables and lifestyle information as well as records of physical and biomedical examinations. Second, this study used ICD codes used by most physicians for stroke diagnosis. Stroke-related clinical risk scores and information on lesion characteristics were not available for analysis of stroke severity. Third, patients diagnosed with stroke before the 24-month period prior to surgery could have been included in the control group and conversely, patients with minor symptoms of stroke without emergency treatment or hospitalization may potentially be missing in case group of patients. Finally, although the 
accuracy of major diagnosis codes in the National Health Insurance Research Database has been studied ${ }^{26}$, the validity of comorbidity and complications determined by using reimbursement codes needs to be verified.

In the context of the limitations of the study, the results of the present investigation show that patients with stroke in the 24 month period prior to surgery have a higher incidence postoperative complications and 30-day mortality.

\section{Acknowledgement}

This study is based in part on data obtained from the National Health Insurance Research Database provided by the Bureau of National Health Insurance, Department of Health and managed by the National Health Research Institutes. The interpretation and conclusions contained herein do not represent those of the Bureau of National Health Insurance, Department of Health or National Health Research Institutes. 


\section{References}

1. Lee S, Shafe AC, Cowie MR. UK stroke incidence, mortality and cardiovascular risk management 1999-2008: time-trend analysis from the General Practice Research Database. BMJ Open 2011; 1: e000269.

2. Strong K, Mathers C, Bonita R. Preventing stroke: saving lives around the world. Lancet Neurol 2007; 6: 182-187.

3. O'Donnell MJ1, Xavier D, Liu L, Zhang H, Chin SL, Rao-Melacini P, Rangarajan S, Islam S, Pais P, McQueen MJ, Mondo C, Damasceno A, Lopez-Jaramillo P, Hankey GJ, Dans AL, Yusoff K, Truelsen T, Diener HC, Sacco RL, Ryglewicz D, Czlonkowska A, Weimar C, Wang X, Yusuf S. Risk factors for ischaemic and intracerebral haemorrhagic stroke in 22 countries (the INTERSTROKE study): a case-control study. Lancet 2010; 376:

112-123.

4. Feigin VL. Stroke in developing countries: can the epidemic be stopped and outcomes improved? Lancet Neurol 2007; 6: 94-97.

5. Chou YC, Liao CC, Su LT, Yang PY, Sung FC. Stroke rehabilitation is associated with a reduction in dementia risk: a population-based retrospective cohort study. J Rehabil Med 2012; 44: 319-324.

6. Kumar S, Selim MH, Caplan LR. Medical complications after stroke. Lancet Neurol 2010; 9: $105-118$. 
7. Langhorne P, Stott DJ, Robertson L, MacDonald J, Jones L, McAlpine C, Dick F, Taylor GS, Murray G. Medical complications after stroke: a multicenter study. Stroke 2000; 31: $1223-1229$.

8. Liao CC, Chou YC, Yeh CC, Hu CJ, Chiu WT, Chen TL. Stroke risk and outcomes in patients with traumatic brain injury: 2 nationwide studies. Mayo Clin Proc 2014; 89: 163-172.

9. Koennecke HC, Belz W, Berfelde D, Endres M, Fitzek S, Hamilton F, Kreitsch P, Mackert BM, Nabavi DG, Nolte CH, Pöhls W, Schmehl I, Schmitz B, von Brevern M, Walter G, Heuschmann PU. Factors influencing in-hospital mortality and morbidity in patients treated on a stroke unit. Neurology 2011; 77: 965-972.

10. Mickleborough LL, Walker PM, Takagi Y, Ohashi M, Ivanov J, Tamariz M. Risk factors for stroke in patients undergoing coronary artery bypass grafting. J Thorac Cardiovasc Surg 1996; 112: 1250-1258.

11. Maithel SK, Pomposelli FB, Williams M, Sheahan MG, Scovell SD, Campbell DR, LoGerfo FW, Hamdan AD. Creatinine clearance but not serum creatinine alone predicts long-term postoperative survival after lower extremity revascularization. Am J Nephrol 2006; 26: 612-620.

12. Mui KW1, Zeebregts CJ, van den Hout H, van Baal JG, Navis G, Jan-Woittiez A. Impact of incidental renal artery stenosis on long-term mortality in patients with peripheral 
arterial disease undergoing vascular procedure. J Vasc Surg 2011; 54: 785-790.

13. Bottle A, Mozid A, Grocott HP, Walters MR, Lees KR, Aylin P, Sanders RD. Preoperative stroke and outcomes after coronary artery bypass graft surgery. Anesthesiology 2013; 118: 885-893.

14. Deiwick M, Tandler R, Möllhoff T, Kerber S, Rötker J, Roeder N, Scheld HH. Heart surgery in patients aged eighty years and above: determinants of morbidity and mortality. Thorac Cardiovasc Surg 1997; 45: 119-126.

15. Hata M, Seevanayagam S, Manson N, Rosalion A, Matalanis G, Raman J, Buxton BF. Radial artery 2000: risk analysis of mortality for coronary bypass surgery with radial artery. Ann Thorac Cardiovasc Surg 2002; 8: 354-357.

16. Gupta PK, Franck C, Miller WJ, Gupta H, Forse RA. Development and validation of a bariatric surgery morbidity risk calculator using the prospective, multicenter NSQIP dataset. J Am Coll Surg 2011; 212: 301-309.

17. Sanders RD, Bottle A, Jameson SS, Mozid A, Aylin P, Edger L, Ma D, Reed MR, Walters M, Lees KR, Maze M. Independent preoperative predictors of outcomes in orthopedic and vascular surgery: the influence of time interval between an acute coronary syndrome or stroke and the operation. Ann Surg 2012; 255: 901-907.

18. Bozic KJ, Lau E, Kurtz S, Ong K, Rubash H, Vail TP, Berry DJ. Patient-related risk factors for periprosthetic joint infection and postoperative mortality following total hip 
arthroplasty in Medicare patients. J Bone Joint Surg Am 2012; 94: 794-800.

19. Bozic KJ, Lau E, Kurtz S, Ong K, Berry DJ. Patient-related risk factors for postoperative mortality and periprosthetic joint infection in medicare patients undergoing TKA. Clin Orthop Relat Res 2012; 470: 130-137.

20. Liao CC, Su TC, Sung FC, Chou WH, Chen TL. Does hepatitis C virus infection increase risk for stroke? A population-based cohort study. PLoS One 2012; 7: e31527.

21. Wang IK, Chang SN, Liao CC, Liang CC, Chang CT, Lin HH, Liu JH, Liu YL, Chuang FR, Hsu CY, Huang CC, Sung FC. Hypertensive disorders in pregnancy and preterm delivery and subsequent stroke in Asian women: a retrospective cohort study. Stroke 2011; 42: 716-721.

22. Hu CJ, Liao CC, Chang CC, Wu CH, Chen TL. Postoperative adverse outcomes in surgical patients with dementia: a retrospective cohort study. World J Surg 2012; 36: 2051-2058.

23. Chang CC, Chang HC, Wu CH, Chang CY, Liao CC, Chen TL. Adverse postoperative outcomes in surgical patients with immune thrombocytopenia. Br J Surg 2013; 100: 684-692.

24. Yeh CC, Liao CC, Chang YC, Jeng LB, Yang HR, Shih CC, Chen TL. Adverse outcomes after noncardiac surgery in patients with diabetes: a nationwide population-based retrospective cohort study. Diabetes Care 2013; 36: 3216-3221. 
25. Liao CC, Shen WW, Chang CC, Chang H, Chen TL. Surgical adverse outcomes in patients with schizophrenia: a population-based study. Ann Surg 2013; 257: 433-438.

26. Cheng CL, Kao YH, Lin SJ, Lee CH, Lai ML. Validation of the National Health Insurance Research Database with ischemic stroke cases in Taiwan. Pharmacoepidemiol Drug Saf 2011; 20: 236-242.

27. Wijeysundera DN, Beattie WS, Austin PC, Hux JE, Laupacis A. Epidural anaesthesia and survival after intermediate-to-high risk non-cardiac surgery: a population-based cohort study. Lancet 2008; 372: 562-569.

28. Wijeysundera DN, Beattie WS, Karkouti K, Neuman MD, Austin PC, Laupacis A. Association of echocardiography before major elective non-cardiac surgery with postoperative survival and length of hospital stay: population based cohort study. $B M J$ 2011; 342: d3695.

29. Wijeysundera DN, Beattie WS, Austin PC, Hux JE, Laupacis A. Non-invasive cardiac stress testing before elective major non-cardiac surgery: population based cohort study. BMJ 2010; 340: b5526.

30. Kudoh A. Perioperative management for chronic schizophrenic patients. Anesth Analg 2005; 101: 1867-1872. 
Suppl. Table 1 Definition of coexisting medical conditions and postoperative complications

\begin{tabular}{llll}
\hline Medical conditions & ICD-9-CM & Complications & ICD-9-CM \\
\hline COPD & $490-496$ & Acute myocardial infarction & 410 \\
Diabetes & 250 & Acute renal failure & 584 \\
Hyperlipidemia & 272.9 & Deep wound infection & 958.3 \\
Hypertension & $401-405$ & Postoperative bleeding & $998.0,998.1,998.2$ \\
Ischemic heart disease & $410-414$ & Pulmonary embolism & 415 \\
Liver cirrhosis & $571.2,571.5$ & Pneumonia & $480-486$ \\
Mental disorders & $290-319$ & Septicemia & $038,998.5$ \\
Renal dialysis* & D8, D9 & & \\
Stroke & $430-438$ & & \\
\hline
\end{tabular}

*Defined by administration code.

COPD, chronic obstructive pulmonary disease; ICD-9-CM, International Classification of Diseases, 9th Revision, Clinical Modification. 
Table 1 Characteristics of the cohort of patients with and without stroke in the 24 month period prior to surgery.

\begin{tabular}{|c|c|c|c|c|c|}
\hline \multirow[b]{2}{*}{ Sex } & \multicolumn{2}{|c|}{$\begin{array}{c}\text { No stroke } \\
\mathrm{n}=1381375\end{array}$} & \multicolumn{2}{|c|}{$\begin{array}{c}\text { Stroke } \\
\mathrm{n}=45420\end{array}$} & \multirow{2}{*}{$\frac{P}{<0.001}$} \\
\hline & $\mathrm{n}$ & $(\%)$ & $\mathrm{n}$ & $(\%)$ & \\
\hline Female & 785615 & $(56.9)$ & 18480 & $(40.7)$ & \\
\hline Male & 595760 & $(43.1)$ & 26940 & $(59.3)$ & \\
\hline Age, years & & & & & $<0.001$ \\
\hline $20-29$ & 218284 & $(15.8)$ & 591 & $(1.3)$ & \\
\hline $30-39$ & 296777 & $(21.5)$ & 1022 & $(2.3)$ & \\
\hline $40-49$ & 264382 & $(19.1)$ & 2602 & $(5.7)$ & \\
\hline $50-59$ & 248939 & $(18.0)$ & 6457 & $(14.2)$ & \\
\hline $60-69$ & 166512 & $(12.1)$ & 9333 & $(20.6)$ & \\
\hline$\geq 70$ & 186481 & $(13.5)$ & 25415 & $(56.0)$ & \\
\hline Operation in teaching hospital & 1174886 & $(85.0)$ & 41068 & $(90.4)$ & $<0.001$ \\
\hline Low income status* & 27810 & $(2.0)$ & 1542 & $(3.4)$ & $<0.001$ \\
\hline Type of surgery & & & & & $<0.001$ \\
\hline Skin & 55583 & $(4.0)$ & 2585 & (5.7) & \\
\hline Breast & 34755 & $(2.5)$ & 376 & $(0.8)$ & \\
\hline Musculoskeletal & 393643 & $(28.5)$ & 17199 & $(37.9)$ & \\
\hline Respiratory & 74484 & $(5.4)$ & 2954 & $(6.5)$ & \\
\hline Cardiovascular & 30940 & $(2.2)$ & 4021 & $(8.9)$ & \\
\hline Digestive & 289747 & $(21.0)$ & 9600 & $(21.1)$ & \\
\hline Kidney, ureter, bladder & 108759 & $(7.9)$ & 4970 & $(10.9)$ & \\
\hline Delivery, CS, abortion & 178778 & $(12.9)$ & 157 & $(0.4)$ & \\
\hline Eye & 14761 & $(1.1)$ & 634 & (1.4) & \\
\hline Others & 199925 & $(14.5)$ & 2924 & $(6.4)$ & \\
\hline Types of anesthesia & & & & & $<0.001$ \\
\hline General & 895034 & $(64.8)$ & 30842 & $(67.9)$ & \\
\hline Epidural or spinal & 486341 & $(35.2)$ & 14578 & $(32.1)$ & \\
\hline \multicolumn{6}{|l|}{ Coexisting medical conditions } \\
\hline Hypertension & 227733 & $(16.5)$ & 20156 & $(44.4)$ & $<0.001$ \\
\hline Diabetes & 119808 & $(8.7)$ & 12999 & $(28.6)$ & $<0.001$ \\
\hline Mental disorders & 174339 & $(12.6)$ & 11521 & $(25.4)$ & $<0.001$ \\
\hline COPD & 132181 & $(9.6)$ & 9413 & $(20.7)$ & $<0.001$ \\
\hline Ischemic heart disease & 58576 & $(4.2)$ & 7223 & $(15.9)$ & $<0.001$ \\
\hline Hyperlipidemia & 71250 & $(5.2)$ & 2947 & $(6.5)$ & $<0.001$ \\
\hline
\end{tabular}




\begin{tabular}{llllll} 
Renal dialysis & 15394 & $(1.1)$ & 1927 & $(4.2)$ & $<0.001$ \\
Liver cirrhosis & 36868 & $(2.7)$ & 1552 & $(3.4)$ & $<0.001$ \\
\hline
\end{tabular}

COPD; chronic obstructive pulmonary disease, CS; caesarian section.

*Defined as patients qualified for waived medical copayment as certified by the Bureau of National Health Insurance. 
Table 2 Characteristics of propensity score-matched patients with and without previous stroke

\begin{tabular}{|c|c|c|c|c|c|}
\hline \multirow[b]{2}{*}{ Sex } & \multicolumn{2}{|c|}{$\begin{array}{c}\text { No stroke } \\
\mathrm{n}=45420\end{array}$} & \multicolumn{2}{|c|}{$\begin{array}{c}\text { Stroke } \\
n=45420\end{array}$} & \multirow{2}{*}{$\begin{array}{c}P \\
1.000\end{array}$} \\
\hline & $\mathrm{n}$ & $(\%)$ & $\mathrm{n}$ & $(\%)$ & \\
\hline Female & 18480 & $(40.7)$ & 18480 & $(40.7)$ & \\
\hline Male & 26940 & $(59.3)$ & 26940 & $(59.3)$ & \\
\hline Age, years & & & & & 1.000 \\
\hline $20-29$ & 591 & $(1.3)$ & 591 & (1.3) & \\
\hline $30-39$ & 1022 & $(2.3)$ & 1022 & (2.3) & \\
\hline $40-49$ & 2602 & $(5.7)$ & 2602 & $(5.7)$ & \\
\hline $50-59$ & 6457 & $(14.2)$ & 6457 & $(14.2)$ & \\
\hline $60-69$ & 9333 & $(20.6)$ & 9333 & $(20.6)$ & \\
\hline$\geq 70$ & 25415 & $(56.0)$ & 25415 & $(56.0)$ & \\
\hline Operation in teaching hospital & 41068 & $(90.4)$ & 41068 & $(90.4)$ & 1.000 \\
\hline Low income status* & 1542 & (3.4) & 1542 & (3.4) & 1.000 \\
\hline Type of surgery & & & & & 1.000 \\
\hline Skin & 2585 & $(5.7)$ & 2585 & (5.7) & \\
\hline Breast & 376 & $(0.8)$ & 376 & $(0.8)$ & \\
\hline Musculoskeletal & 17199 & $(37.9)$ & 17199 & $(37.9)$ & \\
\hline Respiratory & 2954 & $(6.5)$ & 2954 & $(6.5)$ & \\
\hline Cardiovascular & 4021 & $(8.9)$ & 4021 & $(8.9)$ & \\
\hline Digestive & 9600 & $(21.1)$ & 9600 & $(21.1)$ & \\
\hline Kidney, ureter, bladder & 4970 & $(10.9)$ & 4970 & $(10.9)$ & \\
\hline Delivery, CS, abortion & 157 & $(0.4)$ & 157 & $(0.4)$ & \\
\hline Eye & 634 & (1.4) & 634 & (1.4) & \\
\hline Others & 2924 & $(6.4)$ & 2924 & (6.4) & \\
\hline Types of anesthesia & & & & & 1.000 \\
\hline General & 30842 & $(67.9)$ & 30842 & $(67.9)$ & \\
\hline Epidural or spinal & 14578 & $(32.1)$ & 14578 & $(32.1)$ & \\
\hline \multicolumn{6}{|l|}{ Coexisting medical conditions } \\
\hline Hypertension & 20156 & $(44.4)$ & 20156 & $(44.4)$ & 1.000 \\
\hline Diabetes & 12999 & $(28.6)$ & 12999 & $(28.6)$ & 1.000 \\
\hline Mental disorders & 11521 & $(25.4)$ & 11521 & $(25.4)$ & 1.000 \\
\hline COPD & 9413 & $(20.7)$ & 9413 & $(20.7)$ & 1.000 \\
\hline Ischemic heart disease & 7223 & $(15.9)$ & 7223 & $(15.9)$ & 1.000 \\
\hline Hyperlipidemia & 2947 & $(6.5)$ & 2947 & $(6.5)$ & 1.000 \\
\hline Renal dialysis & 1927 & $(4.2)$ & 1927 & $(4.2)$ & 1.000 \\
\hline
\end{tabular}


COPD, chronic obstructive pulmonary disease, CS; caesarian section.

*Defined as patients qualified for waived medical copayment as certified by the Bureau of National Health Insurance. 
Table 3 Postoperative adverse events for patients with preoperative stroke before and after using propensity-score matching methods

\begin{tabular}{lrrrrc}
\hline & \multicolumn{2}{c}{ No stroke } & \multicolumn{2}{c}{ Stroke } & Adjusted* \\
\cline { 2 - 6 } & $\mathrm{n}$ & $(\%)$ & $\mathrm{n}$ & $(\%)$ & RR (95\% c.i.) \\
\hline Before propensity-score matching & & & & & \\
Postoperative complications & & & & & \\
Pneumonia & 15442 & $(1.1)$ & 2365 & $(5.2)$ & $1.86(1.77-1.94)$ \\
Septicemia & 46793 & $(3.4)$ & 5096 & $(11.2)$ & $2.14(2.08-2.21)$ \\
Acute renal failure & 7223 & $(0.5)$ & 1434 & $(3.2)$ & $2.18(2.05-2.31)$ \\
Acute myocardial infarction & 2521 & $(0.2)$ & 487 & $(1.1)$ & $1.47(1.33-1.63)$ \\
Deep wound infection & 7434 & $(0.5)$ & 282 & $(0.6)$ & $1.04(0.92-1.18)$ \\
Pulmonary embolism & 792 & $(0.1)$ & 85 & $(0.2)$ & $1.53(1.21-1.93)$ \\
Postoperative bleeding & 7575 & $(0.6)$ & 358 & $(0.8)$ & $1.05(0.94-1.17)$ \\
Any of the above & 77815 & $(5.6)$ & 8218 & $(18.1)$ & $1.90(1.85-1.94)$ \\
30-day postoperative mortality & 5205 & $(0.4)$ & 956 & $(2.1)$ & $2.41(2.24-2.59)$ \\
Prolonged LOS $\dagger$ & 260747 & $(18.9)$ & 16911 & $(37.2)$ & $1.49(1.46-1.52)$ \\
ICU stay & 312251 & $(22.6)$ & 26240 & $(57.8)$ & $1.77(1.75-1.79)$ \\
Increased medical expenditure $\dagger$ & 268671 & $(19.5)$ & 16328 & $(36.0)$ & $1.19(1.17-1.21)$ \\
After propensity-score matching & & & & & \\
Postoperative complications & & & & & \\
Pneumonia & 1223 & $(2.7)$ & 2365 & $(5.2)$ & $1.93(1.80-2.07)$ \\
Septicemia & 2785 & $(6.1)$ & 5096 & $(11.2)$ & $1.83(1.75-1.92)$ \\
Acute renal failure & 841 & $(1.9)$ & 1434 & $(3.2)$ & $1.71(1.57-1.86)$ \\
Acute myocardial infarction & 372 & $(0.8)$ & 487 & $(1.1)$ & $1.31(1.14-1.50)$ \\
Deep wound infection & 285 & $(0.6)$ & 282 & $(0.6)$ & $0.99(0.84-1.16)$ \\
Pulmonary embolism & 50 & $(0.1)$ & 85 & $(0.2)$ & $1.70(1.20-2.41)$ \\
Postoperative bleeding & 361 & $(0.8)$ & 358 & $(0.8)$ & $0.99(0.86-1.15)$ \\
Any of the above & 4896 & $(10.8)$ & 8218 & $(18.1)$ & $1.68(1.62-1.74)$ \\
30-day postoperative mortality & 538 & $(1.2)$ & 956 & $(2.1)$ & $1.79(1.61-1.99)$ \\
Prolonged LOS $\dagger$ & 7242 & $(15.9)$ & 10141 & $(22.3)$ & $1.40(1.36-1.44)$ \\
ICU stay & 15473 & $(34.1)$ & 19114 & $(42.1)$ & $1.24(1.21-1.26)$ \\
\hline
\end{tabular}

ICU, intensive care unit; LOS, length of stay; c.i., confidence interval; RR, rate ratio.

*Adjusted for age, sex, teaching hospital, low income, urbanization, coexisting medical conditions, type of surgery and type of anesthesia.

$\dagger$ Categorized into quartiles. Surgical patients who had the highest quartile of length of stay or in-hospital medical expenditures were defined as the individuals having 
increased length of stay or elevated medical expenditures. 
Table 4 Adjusted rate ratio and 95 per cent confidence intervals of 30-day mortality associated with stroke-related treatment, characteristics and comorbidities prior to surgery

\begin{tabular}{|c|c|c|c|c|}
\hline \multirow{2}{*}{ Preoperative characteristics for stroke } & \multirow[b]{2}{*}{$\mathrm{n}$} & \multirow[b]{2}{*}{ Deaths } & \multirow[b]{2}{*}{ Mortality, \% } & 30-day mortality \\
\hline & & & & RR $(95 \% \text { c.i. })^{*}$ \\
\hline \multicolumn{5}{|l|}{ Time period for stroke diagnosis } \\
\hline No stroke & 45420 & 538 & 1.2 & 1.00 (reference) \\
\hline Stroke within $13-24$ months preoperatively & 28348 & 424 & 1.5 & $1.25(1.10-1.42)$ \\
\hline Stroke within 7-12 months preoperatively & 6494 & 96 & 1.5 & $1.25(1.00-1.55)$ \\
\hline Stroke within 1-6 months preoperatively & 10578 & 436 & 4.1 & $3.31(2.91-3.75)$ \\
\hline \multicolumn{5}{|l|}{ Low income status $\uparrow$} \\
\hline No stroke & 45420 & 538 & 1.2 & 1.00 (reference) \\
\hline Patients with stroke without low income & 43878 & 913 & 2.1 & $1.77(1.59-1.97)$ \\
\hline Patients with stroke with low income & 1542 & 43 & 2.8 & $2.30(1.68-3.15)$ \\
\hline \multicolumn{5}{|l|}{ Type of stroke } \\
\hline No stroke & 45420 & 538 & 1.2 & 1.00 (reference) \\
\hline Ischemic stroke & 24561 & 414 & 1.7 & $1.42(1.25-1.61)$ \\
\hline Other unclassified stroke & 11862 & 185 & 1.6 & $1.36(1.15-1.61)$ \\
\hline Hemorrhagic stroke & 8997 & 357 & 4.0 & $3.41(2.97-3.91)$ \\
\hline \multicolumn{5}{|l|}{ Preoperative ICU stay } \\
\hline No stroke & 45420 & 538 & 1.2 & 1.00 (reference) \\
\hline Patients with stroke without ICU stay & 32243 & 541 & 1.7 & $1.45(1.29-1.64)$ \\
\hline Patients with stroke with ICU stay & 13177 & 415 & 3.2 & $2.55(2.24-2.90)$ \\
\hline \multicolumn{5}{|l|}{ Stroke related neurosurgery } \\
\hline No stroke & 45420 & 538 & 1.2 & 1.00 (reference) \\
\hline Patients with stroke and without & 39378 & 743 & 1.9 & $1.61(1.44-1.80)$ \\
\hline \multicolumn{5}{|l|}{ neurosurgery } \\
\hline Patients with stroke and with neurosurgery & 6042 & 213 & 3.5 & $2.49(2.12-2.92)$ \\
\hline \multicolumn{5}{|l|}{ Stroke-related medical expenditure } \\
\hline No stroke & 45420 & 538 & 1.2 & 1.00 (reference) \\
\hline Patients with stroke and very low income & 9086 & 134 & 1.5 & $1.31(1.08-1.59)$ \\
\hline Patients with stroke and low income & 9083 & 146 & 1.6 & $1.39(1.16-1.67)$ \\
\hline Patients with stroke and moderate income & 9083 & 145 & 1.6 & $1.36(1.13-1.63)$ \\
\hline Patients with stroke and high income & 9084 & 239 & 2.6 & $2.19(1.88-2.55)$ \\
\hline Patients with stroke and very high income & 9084 & 292 & 3.2 & $2.35(2.04-2.71)$ \\
\hline \multicolumn{5}{|l|}{ Preoperative TBI } \\
\hline No stroke & 45420 & 538 & 1.2 & 1.00 (reference) \\
\hline Patients with stroke and without TBI & 36836 & 745 & 2.0 & $1.68(1.51-1.88)$ \\
\hline
\end{tabular}


Patients with stroke and with TBI

Preoperative dementia

No stroke

Patients with stroke and without dementia

Patients with stroke and with dementia

\section{Preoperative pneumonia}

No stroke

Patients with stroke and without pneumonia

Patients with stroke and with pneumonia

Preoperative decubitus ulcer

No stroke

Patients with stroke and without decubitus

ulcer

Patients with stroke and with decubitus ulcer
$45420 \quad 538$

$41932 \quad 849$

$45420 \quad 538$

1.21 .00 (reference)

$38429 \quad 780$

$2.0 \quad 1.68$ (1.51-1.88)

$6991 \quad 176$

$2.5 \quad 2.10(1.75-2.51)$

$45420 \quad 538$

1.21 .00 (reference)

$32069 \quad 565$

1.81 .54 (1.37-1.74)

$13351 \quad 391$

$2.92 .16(1.89-2.46)$

1.21 .00 (reference)

$2.0 \quad 1.69(1.52-1.88)$

ICU, intensive care unit; RR, rate ratio; c.i., confidence interval; TBI, traumatic brain injury.

*Adjusted for age, sex, teaching hospital, low income, urbanization, coexisting medical conditions, type of surgery and anesthesia.

$\dagger$ Defined as patients qualified for waived medical copayment as certified by the Bureau of National Health Insurance. 